\title{
RESEARCH
}

Open Access

\section{Synthesis and in vitro antimicrobial activity of new steroidal hydrazone derivatives}

\author{
Shailesh Mistry ${ }^{*}$ (D) and Akhilesh Kumar Singh
}

\section{Abstract}

Background: For many years, various drugs have been used for the treatment of infectious diseases but some bacterial microorganisms have induced resistance to several drugs. In a search of new antimicrobial agents, a series of new steroidal hydrazones were designed and synthesized.

Result: The structures of the compounds were established based on the spectral data. The in vitro antimicrobial activity of some newly synthesized compounds against bacteria and fungi was studied.

Conclusion: New compounds showed better or similar antimicrobial activity. Designing more efficient steroidal hydrazones from ketosteroid based on the current study may successfully lead to the development of antimicrobial agent.

Keywords: Androstene, Estrane, Hydralazine hydrochloride, Hydrazone, Antimicrobial activity

\section{Graphical abstract}

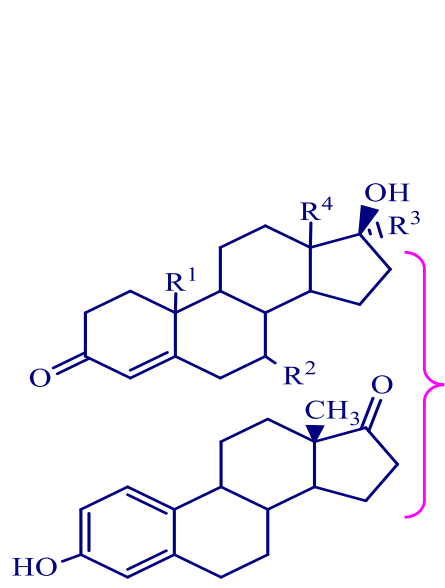

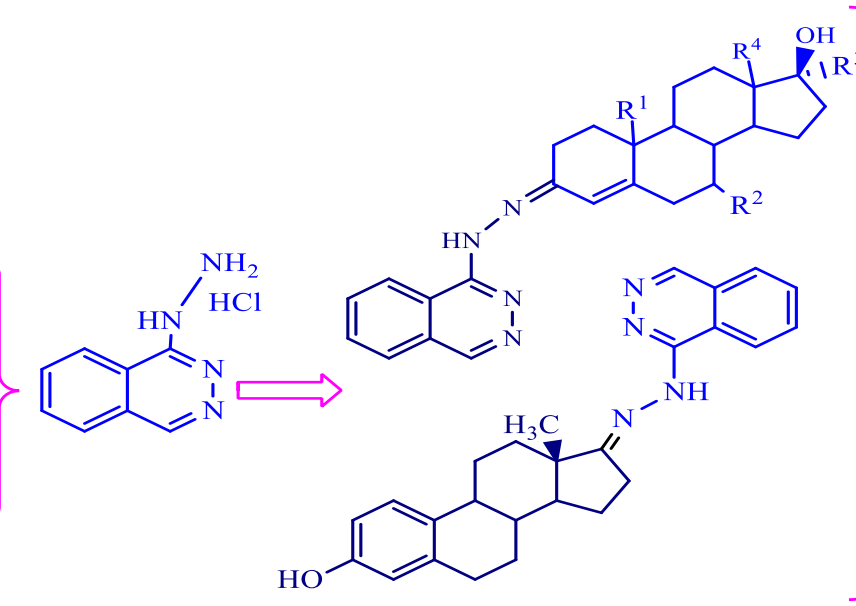

Antimicrobial activity $\left(\mathrm{Gram}^{+}\right.$ve \& Gram$^{-} v e$ bacteria)

*Correspondence: shaileshmistry1017@yahoo.com

Parul Institute of Applied Science, Parul University, P.O. Limbda, Ta.

Waghodiya, Vadodara, Gujarat 391760, India original author(s) and the source, provide a link to the Creative Commons licence, and indicate if changes were made. The images or other third party material in this article are included in the article's Creative Commons licence, unless indicated otherwise in a credit line to the material. If material is not included in the article's Creative Commons licence and your intended use is not permitted by statutory regulation or exceeds the permitted use, you will need to obtain permission directly from the copyright holder. To view a copy of this licence, visit http://creativecommons.org/licenses/by/4.0/. 


\section{Background}

Hydrazones are synthesized by condensation of aldehyde/ketone with hydrazine. Hydrazones are also synthesized by coupling reaction of aryl diazonium salts with active hydrogen compounds [1]. Hydrazone have gained great importance due to their diverse biological properties including antibacterial and antifungal [2], anticonvulsant [3], anti-inflammatory [4], antimalarial [5] and antituberculosis [6] activities. When they are used as intermediates, coupling products can be synthesized by using the active hydrogen component of azomethine group [7].

Searching for new molecules in the field of steroid will never end. Researchers are always been interested to do research on steroid due to its particular biological and pharmacological action. The Steroidal drugs have been widely used in traditional medicines. The versatile activity of androstene and estrane series indicates that these molecules could be a key starting material for developing a new drug. Particularly, this invention relates to therapeutically valuable steroids of androstene and estrane series having hydrazone function. Steroidal hydrazones have received extensive attention of scientists because they exhibit some biological activities such as antifungal, antibacterial, antiproliferative, antituberculosis, antiviral and anticancer [8-16].

Structural modification of steroids requires great synthetic effort and still a vivacious area of research. Steroidal ring modification and incorporation of heteroatom or replacing one or more carbon atoms in steroidal molecule may improves its biological activities have been researched and reported [17-26]. About preparation of steroidal derivatives, introduction of methyl group at a certain position of steroids may significantly change their bioactivities [27]. The investigation of new steroidal derivatives has been given great attention. Hydralazine plays an important role as antihypertensive drug and sold under the brand name Apresolin. Hydralazine belongs to the hydrazinophthalazine class of drugs [28]. Hydralazine derivatives have wide applications in the treatment of diseases such as tuberculosis, mental disorder [29]. Hydralazine can be used as antimicrobial, antihypertensive, antimalarial and antitumoral agents [30-32].

Steroidal hydrazone containing nitrogen atom has been synthesized with the aim of improving selectivity. However steroidal hydrazones with hydralazine hydrochloride were rarely reported. We decided to further explore the antimicrobial properties of steroidal hydrazone by synthesizing new analogs with suitable structural modifications (Fig. 1).

\section{Methods}

All the chemicals were used as received from commercial sources. All reaction progress were monitored by thinlayer chromatography (TLC) analysis using silica gel 60 $\mathrm{F}_{254}$ TLC plates. The melting point was determined on a Veego-matic melting point apparatus. IR spectra were recorded using potassium bromide disks on a Shimadzo IR Affinity 1S. The wave numbers are given in $\mathrm{cm}^{-1}$. ${ }^{1} \mathrm{H}$ and $C^{13}$ NMR spectra were recorded on Bruker Avance II spectrophotometer at 400 and $300 \mathrm{MHz}$ and 100 and $75 \mathrm{MHz}$, respectively, with tetramethylsilane as an internal reference; the chemical shifts were measured in ppm with respect to the solvent. Mass spectra were recorded on TSQ Quantum and water make Acquity model UPLC connected with SQ detector (Single Quadra pole) software Mass Lynx (401) instrument equipped with electro

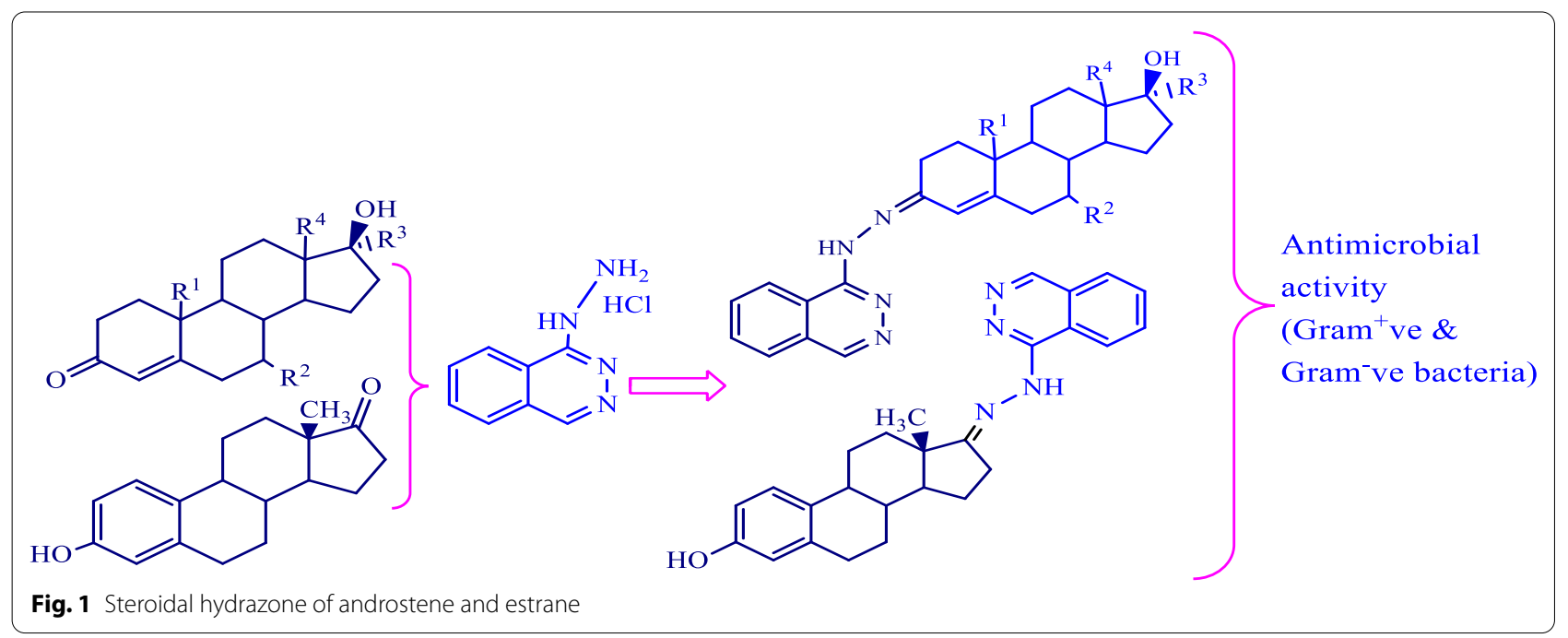




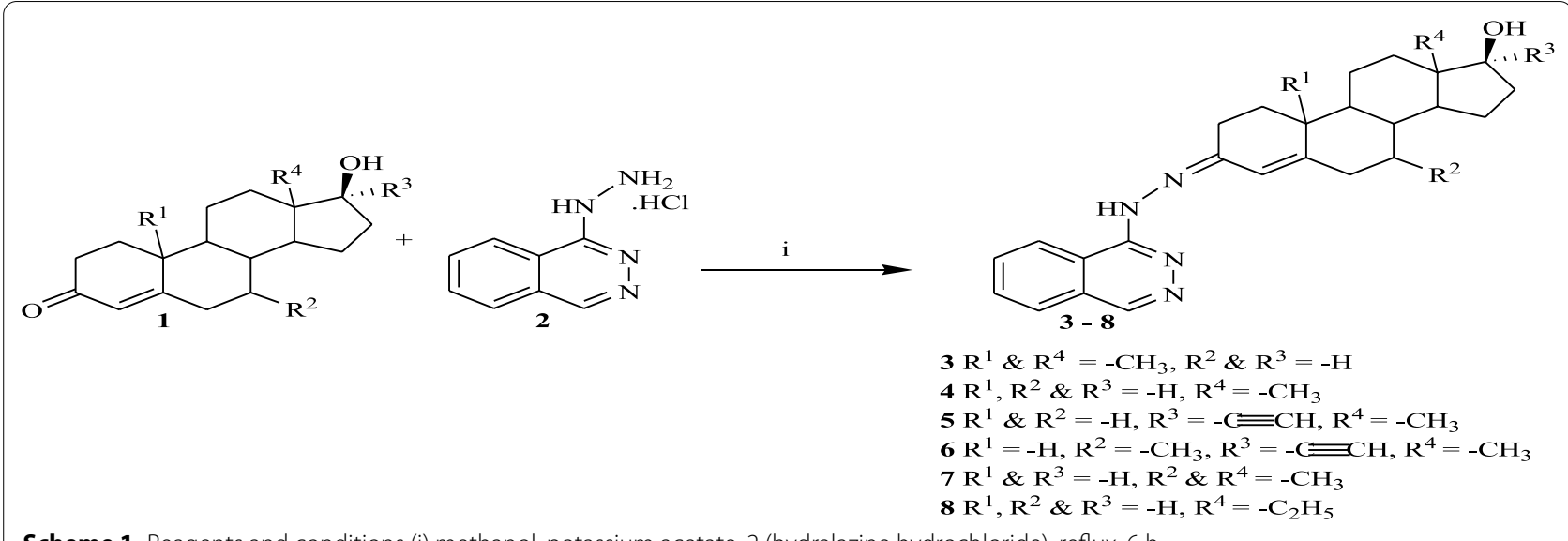

Scheme 1 Reagents and conditions (i) methanol, potassium acetate, 2 (hydralazine hydrochloride), reflux, $6 \mathrm{~h}$

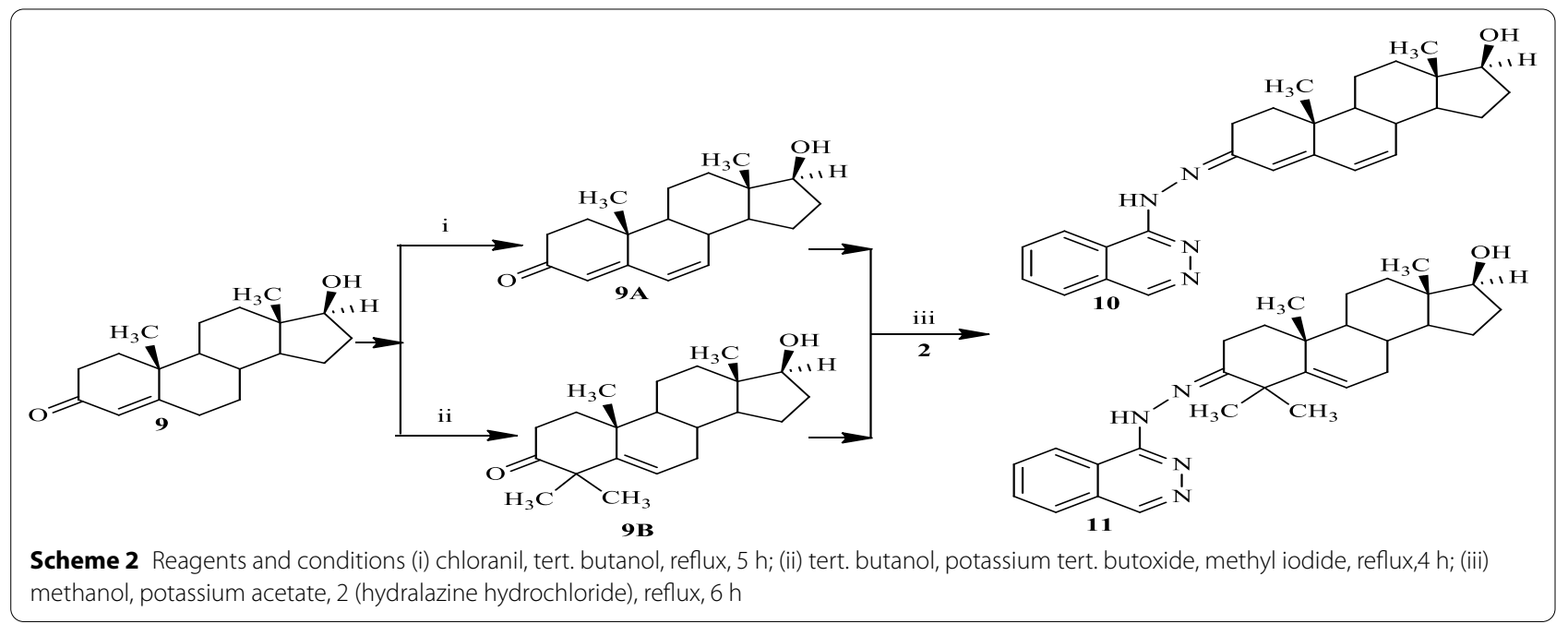

spray ionization (ESI) ion source. Measurements were taken in positive (MS+) ion mode.

\section{Experimental}

\section{General procedure for the preparation of steroidal}

hydrazone $(3-8,10,11,15,16,18,20$ and 22)

Ketosteroid (1, 9A, 9B, 14A, 14B, 17A, 19 and 21) (Schemes 1, 2, 4, 5, 6, 7) (2.5 mmol) and hydralazine hydrochloride (2) $(2.6 \mathrm{mmol})$ with Potassium acetate $(2.6 \mathrm{mmol})$ in Methanol $(25 \mathrm{ml})$ was refluxed for $6 \mathrm{~h}$. After the end of the reaction (monitored by TLC), the mixture was allowed to cool and added water $(25 \mathrm{ml})$. On stirring, the precipitate was formed and collected by filtration. This solid was purified from Methanol $(10 \mathrm{ml})$ and dried at $45-50{ }^{\circ} \mathrm{C}$ to afford the corresponding compounds as yellow solid.

\section{Synthetic procedure of 9A ( $\Delta 6$-testosterone)}

To a solution of Testosterone (9) (2.0 g) in tert. butanol $(20 \mathrm{ml})$ was added chloranil $(1.6 \mathrm{~g})$ and the reaction mixture was heated to $80{ }^{\circ} \mathrm{C}$ for $5 \mathrm{~h}$. After cooling, the reaction mixture was poured into $10 \% \mathrm{Na}_{2} \mathrm{CO}_{3}$, solution and the products were extracted with methylene dichloride. The extracts were washed with water dried over anhydrous sodium sulfate and the solvent was evaporated to afford crude crystals. Recrystallization from acetone gave $(1.0 \mathrm{~g}) 9 \mathrm{~A}$.

\section{Synthetic procedure of 9B}

$5 \mathrm{~g}$ of Testosterone (9) in tert. butanol (50 ml) was stirred under nitrogen and charged potassium tert. butoxide ( $5 \mathrm{~g}$ ) in the mixture. Stirred the reaction mass until clear solution obtained. Added drop wise solution 
of methyl iodide $(7.0 \mathrm{ml})$ in to the reaction mass at $25-30{ }^{\circ} \mathrm{C}$. Reaction mass allowed to stirred for $4 \mathrm{~h}$ (monitored by TLC) at $25-30{ }^{\circ} \mathrm{C}$. Water $(100 \mathrm{ml})$ was added, the tert-butanol removed in vacuum and cooled the suspended mass and filtered. Recrystallization from acetone to give compound (2.5 g) 9B. MS (ESI+) $m / z$ : Calculated for $\mathrm{C}_{21} \mathrm{H}_{32} \mathrm{O}_{2}[\mathrm{M}+\mathrm{H}]^{+} 316.48$; found, 317.31.

\section{Method for the preparation of compound 10 and 11 according to the general procedure}

See Scheme 2 .

\section{Synthetic procedure of (13)}

Estrone (12) (0.75 g, $2.8 \mathrm{~mol})$ and hydralazine hydrochloride (2) (0.58 g, $2.9 \mathrm{~mol})$ with Potassium acetate $(0.28 \mathrm{~g}, 2.8 \mathrm{~mol})$ in Tetrahydrofuran $(25 \mathrm{ml})$ was refluxed for $8-10 \mathrm{~h}$. After the end of the reaction (monitored by TLC), Distilled off Tetrahydrofuran under reduced pressure and yellowish oily mass allowed to cool and added Methanol (15 ml). On stirring, the precipitate was formed and collected by filtration and washed with water $(20 \mathrm{ml})$. This solid was purified from Methanol $(10 \mathrm{ml})$ and dried at $45-50{ }^{\circ} \mathrm{C}$ to afford the corresponding compounds (13) as yellow solid (Scheme 3 ).

\section{Synthetic procedure of 14A (4-methyl nandrolone) and 14B}

To a solution of Nandrolone (14) in tert. butanol was added potassium tert-butoxide with stirring under inert atmosphere by nitrogen blanketing. The solution of methyl iodide $(3.8 \mathrm{ml}, 61.04 \mathrm{mmol})$ in tert-butanol $(18 \mathrm{ml})$ was added drop wise over a period of $30 \mathrm{~min}$. and the resulting mixture was refluxed for $30 \mathrm{~min}$ (monitored by TLC). After cooling, the reaction mixture was acidified with $1 \mathrm{M} \mathrm{HCl}$ and the solvent was evaporated. The crude product was extracted with Methylene dichloride, the organic layer was washed with $\mathrm{NaHSO}_{3}$ and water, dried with anhydrous sodium sulfate and distilled out solvent under vacuum to give crude product which was separated by flash chromatography over short silica column eluting with $30 \%$ ethyl acetate in $n$-Hexane to give white solid of 4-methyl-androst-4-en-3-one-17ß-ol (14A) MS $(\mathrm{ESI}+) \mathrm{m} / z$ : Calculated for $\mathrm{C}_{19} \mathrm{H}_{28} \mathrm{O}_{2}[\mathrm{M}+\mathrm{H}]^{+}$288.42;<smiles>CC1(C)C(=O)CCC1C1CCC2C1CCC1C2CCC2C3CCc4cc(O)ccc4C3CC[C@@]21C</smiles>

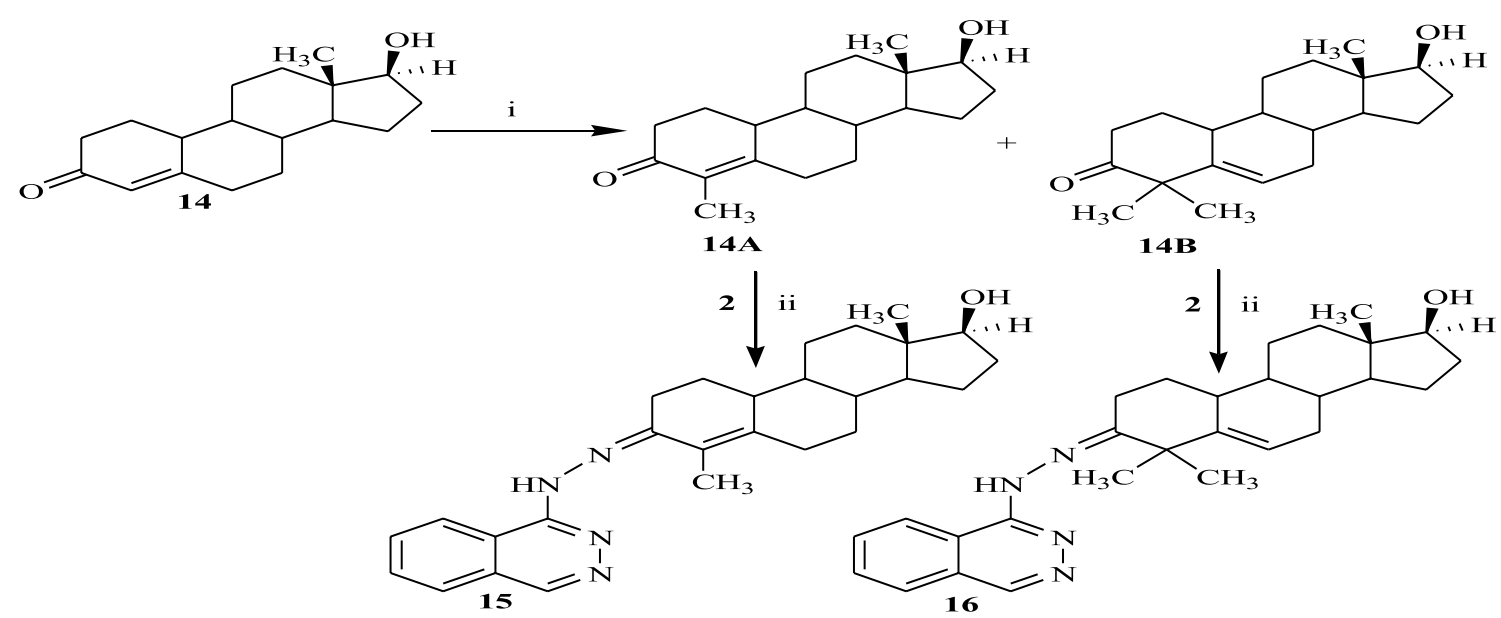

Scheme 4 Reagents and conditions (i) tert-butanol, potassium tert-butoxide, methyl iodide, reflux, 1 h; (ii) methanol, potassium acetate, 2 (hydralazine hydrochloride), reflux, $6 \mathrm{~h}$ 
found, 289.16 and 4,4-dimethyl-androst-5-en-3-one$17 \beta$-ol(14B) MS (ESI+) $m / z$ : Calculated for $\mathrm{C}_{20} \mathrm{H}_{30} \mathrm{O}_{2}$ $[\mathrm{M}+\mathrm{H}]^{+}$302.45; found, 303.21.

Method for the preparation of compound 15 and 16 according to the general procedure

See Scheme 4.
Synthetic procedure of $17 \mathrm{~A}$ ( $\Delta 6$-norethisterone) according to the procedure $9 \mathrm{~A}$ and method for the preparation of compound 18 according to the general procedure See Scheme 5.

\section{Method for the preparation of compound 20 according} to the general procedure

See Scheme 6.<smiles>C#C[C@]1(O)CCC2C3C=CC4=CC(=NNc5nncc6ccccc56)CCC4C3CC[C@]2(C)C1CC#CC</smiles>

Scheme 5 Reagents and conditions (i) Chloranil, tert-butanol, reflux, 5 h; (ii) methanol, potassium acetate (2) hydralazine hydrochloride, reflux, $6 \mathrm{~h}$

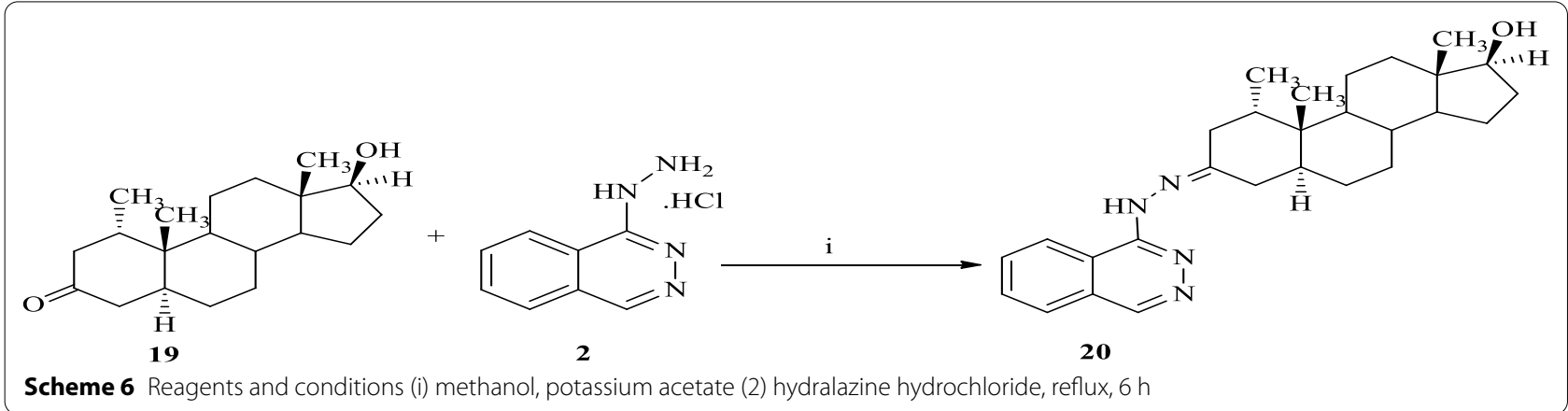

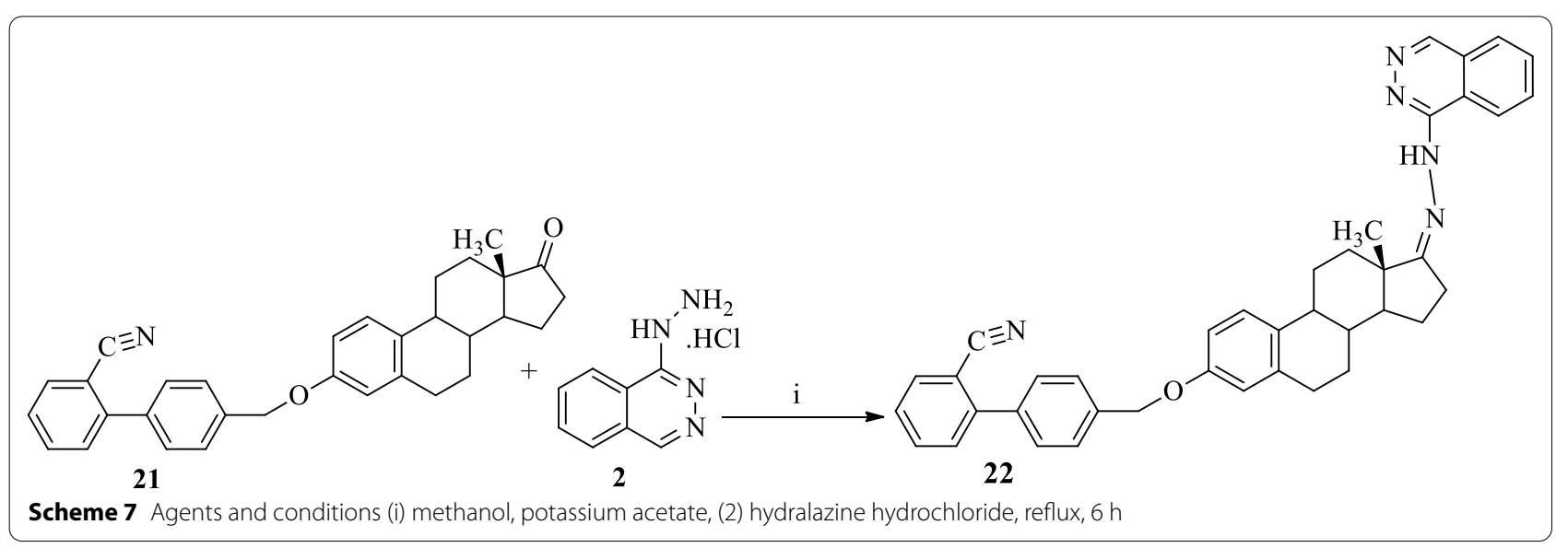




\author{
Method for the preparation of compound 22 according \\ to the general procedure \\ See Scheme 7.
}

\section{Results}

3-(Phthalazin-1yl-hydrazono)-4-androstene-17 $\beta$-ol (3) Yellow solid, 0.63 g, Yield 67\%; mp: $190{ }^{\circ} \mathrm{C}$ Dec.; IR $\left(\mathrm{KBr}, \mathrm{cm}^{-1}\right)$ : $1582(\mathrm{C}=\mathrm{C}), 1605(\mathrm{C}=\mathrm{N}), 2934(\mathrm{CH})$, 3387( $\mathrm{NH}), 3410(\mathrm{OH}) ;{ }^{1} \mathrm{H}$ NMR (400 $\left.\mathrm{MHz} \mathrm{CDCl}_{3}\right) \delta$, ppm: $0.76\left(\mathrm{~s}, 3 \mathrm{H},-\mathrm{CH}_{3}\right), 1.17\left(\mathrm{~s}, 3 \mathrm{H},-\mathrm{CH}_{3}\right), 3.62(\mathrm{~s}, 1 \mathrm{H}$, $-\mathrm{CH}), 5.70(\mathrm{~s}, 1 \mathrm{H},-\mathrm{CH}) 7.51(\mathrm{~m}, 1 \mathrm{H}, \mathrm{Ar}-\mathrm{H}), 7.67(\mathrm{~m}$, $2 \mathrm{H}, \mathrm{Ar}-\mathrm{H}), 7.86(\mathrm{~s}, 1 \mathrm{H}, \mathrm{Ar}-\mathrm{H}), 8.30(\mathrm{~m}, 1 \mathrm{H}, \mathrm{Ar}-\mathrm{H}) ;{ }^{13} \mathrm{C}$ NMR (100 MHz $\mathrm{CDCl}_{3}$ ): 11.0, 17.4, 20.6, 23.3, 30.3, 31.5, $32.8,33.9,35.6,35.7,36.4,42.8,50.4,53.9,81.4,123.7$, $125.8,126.9,127.3,128.7,131.4,131.7,137.5,146.9$, 152.0, 161.4; MS (ESI+) $m / z$ : calculated for $\mathbf{C}_{27} \mathbf{H}_{34} \mathbf{N}_{\mathbf{4}} \mathbf{O}$ $[\mathrm{M}+\mathrm{H}]^{+}$430.27; found, 431.12 .

3-(Phthalazin-1yl-hydrazono)-19-nor-4-androstene-17 $\beta$-ol (4) Yellow solid, $0.70 \mathrm{~g}$, Yield $62.5 \%$; mp: $>200{ }^{\circ} \mathrm{C}$; IR $\left(\mathrm{KBr}, \mathrm{cm}^{-1}\right): 1590(\mathrm{C}=\mathrm{C}), \quad 1612(\mathrm{C}=\mathrm{N}), 2912(\mathrm{CH})$, 3352(NH), $3409(\mathrm{OH}) ;{ }^{1} \mathrm{H}$ NMR (400 MHz $\left.\mathrm{CDCl}_{3}\right) \delta$, ppm: 0.76(s, 3H, $\left.-\mathrm{CH}_{3}\right), 3.61(\mathrm{~s}, 1 \mathrm{H},-\mathrm{CH}), 5.78(\mathrm{~s}, 1 \mathrm{H},-$ $\mathrm{CH}), 7.71(\mathrm{~m}, 1 \mathrm{H}, \mathrm{Ar}-\mathrm{H}), 7.81(\mathrm{~m}, 2 \mathrm{H}, \mathrm{Ar}-\mathrm{H}), 8.29(\mathrm{~s}, 1 \mathrm{H}$, $\mathrm{Ar}-\mathrm{H})$, 8.51(m, 1H, Ar-H); ${ }^{13} \mathrm{C}$ NMR (100 $\left.\mathrm{MHz} \mathrm{CDCl}_{3}\right)$ : $11.1,23.1,26.1,26.5,30.3,30.6,35.4,36.4,40.4,42.5$, 43.0, 49.5, 49.7, 81.5, 122.9, 124.4, 126.2, 127.5, 128.1, 131.2, 137.6, 145.8, 166.9; MS (ESI+) $m / z$ : Calculated for $\mathrm{C}_{26} \mathbf{H}_{32} \mathbf{N}_{4} \mathrm{O}[\mathrm{M}+\mathrm{H}]^{+}$416.26; found, 417.28.

17-Ethinyl-3-(phthalazin-1yl-hydrazono)-19-nor-4-and rostene-17 $\beta$-ol (5) Yellow solid, 0.65 g, Yield 60\%; mp: 165-167 ${ }^{\circ} \mathrm{C}$; IR $\left(\mathrm{KBr}, \mathrm{cm}^{-1}\right): 1585(\mathrm{C}=\mathrm{C}), 1615(\mathrm{C}=\mathrm{N})$, 2956(CH), 3271( $=\mathrm{C}-\mathrm{H}), 3331(\mathrm{OH}) ;{ }^{1} \mathrm{H}$ NMR $(400 \mathrm{MHz}$ $\left.\mathrm{CDCl}_{3}\right) \delta$, ppm: 0.97(s, 3H, $\left.-\mathrm{CH}_{3}\right), 2.86(\mathrm{~s}, 1 \mathrm{H},-\mathrm{CH})$, 5.73(s, $1 \mathrm{H},-\mathrm{CH}), 7.75(\mathrm{~m}, 1 \mathrm{H}, \mathrm{Ar}-\mathrm{H}), 7.92(\mathrm{~m}, 2 \mathrm{H}$, Ar-H), 8.30(s, 1H, Ar-H), 8.45(m, $1 \mathrm{H}, \mathrm{Ar}-\mathrm{H}) ;{ }^{13} \mathrm{C}$ NMR $\left(100 \mathrm{MHz} \mathrm{CDCl}_{3}\right):$ 11.9, 23.1, 26.4, 26.8, 30.8, 32.6, 35.6, 36.7, 39.0, 41.2, 42.7, 47.0, 49.3, 74.3, 79.8, 87.6, 124.7, $125.9,126.4,127.3,129.1,131.9,132.7,135.5,141.7$, 158.5, 165.8; MS (ESI+) $m / z$ : Calculated for $\mathbf{C}_{28} \mathbf{H}_{32} \mathbf{N}_{\mathbf{4}} \mathbf{O}$ $[\mathrm{M}+\mathrm{H}]^{+}$440.58; found, 441.38 .

17-Ethinyl-3-(phthalazin-1yl-hydrazono)-7 $\alpha$-methyl19-nor-4-androstene-17ß-ol (6) Yellow solid, $0.73 \mathrm{~g}$, Yield 67\%; mp: $>200{ }^{\circ} \mathrm{C}$; IR $\left(\mathrm{KBr}, \mathrm{cm}^{-1}\right): 1580(\mathrm{C}=\mathrm{C})$, 1608(C=N), 2934(CH), 3251 (三C-H), 3404(OH); ${ }^{1} \mathrm{H}$ NMR (400 MHz $\mathrm{CDCl}_{3}$ ) $\delta$, ppm: 0.75(d, $3 \mathrm{H}, J=7.2 \mathrm{~Hz}$, $\left.-\mathrm{CH}_{3}\right), 0.90\left(\mathrm{~s}, 3 \mathrm{H},-\mathrm{CH}_{3}\right), 2.56(\mathrm{~s}, 1 \mathrm{H},-\mathrm{CH}), 5.82(\mathrm{~s}, 1 \mathrm{H},-$ $\mathrm{CH}), 7.75(\mathrm{~m}, 1 \mathrm{H}, \mathrm{Ar}-\mathrm{H}), 7.92(\mathrm{~m}, 2 \mathrm{H}, \mathrm{Ar}-\mathrm{H}), 8.33(\mathrm{~s}, 1 \mathrm{H}$, $\mathrm{Ar}-\mathrm{H}), 8.69(\mathrm{~m}, 1 \mathrm{H}, \mathrm{Ar}-\mathrm{H}) ;{ }^{13} \mathrm{C} \mathrm{NMR}\left(100 \mathrm{MHz} \mathrm{CDCl}_{3}\right)$ : 12.6, 12.8, 22.2, 26.7, 30.6, 32.3, 36.6, 38.7, 42.0, 43.2,
43.4 45.9, 46.9, 74.1, 78.6, 87.3, 124.1, 125.2, 126.7, 127.6, 128.9, 131.5, 132.8, 135.9, 141.3, 157.9, 165.0; MS (ESI+) $m / z$ : Calculated for $\mathbf{C}_{29} \mathbf{H}_{34} \mathbf{N}_{4} \mathbf{O}[\mathrm{M}+\mathrm{H}]^{+}$454.27; found, 455.19 .

7 $\alpha$-Methyl-3-(phthalazin-1yl-hydrazono)-19-nor-4-a ndrostene-17ß-ol (7) Yellow solid, $0.75 \mathrm{~g}$, Yield 66\%; $\mathrm{mp}:>210{ }^{\circ} \mathrm{C}$; IR $\left(\mathrm{KBr}, \mathrm{cm}^{-1}\right): 1587(\mathrm{C}=\mathrm{C}), 1610(\mathrm{C}=\mathrm{N})$, 2927(CH), 3362(NH), 3410(OH); ${ }^{1} \mathrm{H}$ NMR $(400 \mathrm{MHz}$ $\left.\mathrm{CDCl}_{3}\right) \delta$, ppm: 0.73(d, $\left.3 \mathrm{H}, J=8 \mathrm{~Hz},-\mathrm{CH}_{3}\right), 0.90(\mathrm{~s}, 3 \mathrm{H},-$ $\left.\mathrm{CH}_{3}\right), 3.61(\mathrm{~s}, 1 \mathrm{H},-\mathrm{CH}), 5.72(\mathrm{~s}, 1 \mathrm{H},-\mathrm{CH}), 7.71(\mathrm{~m}, 1 \mathrm{H}, \mathrm{Ar}-$ $\mathrm{H}), \quad 7.97(\mathrm{~m}, 2 \mathrm{H}, \mathrm{Ar}-\mathrm{H}), \quad 8.35(\mathrm{~s}, 1 \mathrm{H}, \mathrm{Ar}-\mathrm{H}), 8.67(\mathrm{~m}, 1 \mathrm{H}, \mathrm{Ar}-$ $\mathrm{H}) ;{ }^{13} \mathrm{C} \mathrm{NMR}\left(100 \mathrm{MHzCDCl}_{3}\right): 11.8,12.9,22.5,26.9$, $30.4,32.9,36.9,38.4,42.1,43.3,43.9,45.4,46.3,81.6$, $123.9,125.3,126.5,127.8,128.3,131.7,132.6,135.4$, 141.2, 156.9, 163.7; MS (ESI+) $m / z$ : calculated for $\mathbf{C}_{27} \mathbf{H}_{34} \mathbf{N}_{4} \mathbf{O}[\mathrm{M}+\mathrm{H}]^{+}$430.27; found, 431.22. Anal. Calc. C:75.31, H:7.96, N:13.01; found C:75.24, H:7.59, N:13.13.

18-Methyl-3-(phthalazin-1yl-hydrazono)-19-nor-4-an drostene-17 $\beta$-ol (8) Yellow solid, $0.77 \mathrm{~g}$, Yield 71.9\%; mp: $>200{ }^{\circ} \mathrm{C}$; IR $\left(\mathrm{KBr}, \mathrm{cm}^{-1}\right): 1575(\mathrm{C}=\mathrm{C}), 1609(\mathrm{C}=\mathrm{N})$, 2923(CH), 3393(OH); ${ }^{1} \mathrm{H}$ NMR (400 $\left.\mathrm{MHz} \mathrm{CDCl}_{3}\right) \delta$, ppm: $0.81\left(\mathrm{t}, 3 \mathrm{H}, J=7.1 \mathrm{~Hz},-\mathrm{CH}_{3}\right), 1.20\left(\mathrm{~m}, 2 \mathrm{H},-\mathrm{CH}_{2}\right)$, 3.48(s,1H,-CH), 5.73(s,1H,-CH), 7.76(m, 1H, Ar-H), 7.93(m, 2H, Ar-H), 8.33(s, 1H, Ar-H), 8.49(m, 1H, Ar$\mathrm{H}) ;{ }^{13} \mathrm{C}$ NMR $\left(100 \mathrm{MHz} \mathrm{CDCl}_{3}\right): 8.9,18.6,21.5,26.0$, 28.0, 30.4, 35.2, 35.8, 38.7, 40.5, 42.2, 47.5, 48.6, 50.2, 80.2, 123.6, 124.1, 125.9, 127.0, 128.2, 131.4, 137.3, 145.2, 168.5; MS (ESI+) $m / z$ : Calculated for $\mathbf{C}_{27} \mathbf{H}_{34} \mathbf{N}_{\mathbf{4}} \mathbf{O}$ $[\mathrm{M}+\mathrm{H}]^{+}$430.59; found, 431.44 .

3-(Phthalazin-1yl-hydrazono)-androsta-4,6-dien-17ß-ol (10) Yellow solid, 0.66 g, Yield 58.9\%; mp: $>200{ }^{\circ} \mathrm{C}$;IR $\left(\mathrm{KBr}, \quad \mathrm{cm}^{-1}\right): 1587(\mathrm{C}=\mathrm{C}), \quad 1606(\mathrm{C}=\mathrm{N}), \quad 2933(\mathrm{CH})$, 3381(NH), 3456(OH); ${ }^{1} \mathrm{H}$ NMR (400 $\left.\mathrm{MHz} \mathrm{CDCl}_{3}\right) \delta$, ppm: 0.74(s, $\left.3 \mathrm{H},-\mathrm{CH}_{3}\right), 0.94\left(\mathrm{~s}, 3 \mathrm{H},-\mathrm{CH}_{3}\right), 3.55(\mathrm{t}, 1 \mathrm{H}$, $J=16 \mathrm{~Hz},-\mathrm{CH}), 5.70(\mathrm{~s}, 1 \mathrm{H},-\mathrm{CH}), 5.98(\mathrm{~s}, 1 \mathrm{H},-\mathrm{CH})$, 7.34-7.38(m, 1H, Ar-H), 7.49-7.53(m, 2H, Ar-H), 7.68 (s, $1 \mathrm{H}, \mathrm{Ar}-\mathrm{H}), 8.25-8.30(\mathrm{~m}, 1 \mathrm{H}, \mathrm{Ar}-\mathrm{H}) ;{ }^{13} \mathrm{C}$ NMR (100 $\mathrm{MHz} \mathrm{CDCl}_{3}$ ): 11.0, 16.8, 20.5, 22.3, 23.0, 30.3, 33.2, 36.1, 36.5, 37.2, 43.7, 48.7, 50.9, 81.3, 124.1, 124.2, 125.9, $127.2,127.5,128.6,131.5,131.6,133.8,137.8,146.5,1$ 52.0, 161.6; MS (ESI+) $\mathrm{m} / z$ : Calculated for $\mathbf{C}_{27} \mathbf{H}_{32} \mathbf{N}_{\mathbf{4}} \mathbf{O}$ $[\mathrm{M}+\mathrm{H}]^{+}$428.26; found, 429. 4. Anal. Calc. C:75.67, $\mathrm{H}: 7.53, \mathrm{~N}: 13.07$; found (C:74.29, H:8.15, N:12.19).

3-(Phthalazin-1yl-hydrazono)-4,4'-dimethyl-5-andros tene-17ß-ol (11) Yellow solid, $0.6 \mathrm{~g}$, Yield 55.0\%; mp: 208-210 ${ }^{\circ} \mathrm{C}$; IR $\left(\mathrm{KBr}, \mathrm{cm}^{-1}\right): 1584(\mathrm{C}=\mathrm{C}), 1607(\mathrm{C}=\mathrm{N})$, 2931(CH), 3367(NH), $3426(\mathrm{OH}) ;{ }^{1} \mathrm{H}$ NMR (400 MHz $\left.\mathrm{CDCl}_{3}\right) \delta$, ppm: $0.72\left(\mathrm{~s}, 3 \mathrm{H},-\mathrm{CH}_{3}\right), 0.96\left(\mathrm{~s}, 3 \mathrm{H},-\mathrm{CH}_{3}\right)$, $1.22\left(\mathrm{~s}, 6 \mathrm{H},-\mathrm{CH}_{3}\right), 3.67(\mathrm{~d}, 1 \mathrm{H}, J=8.03 \mathrm{~Hz},-\mathrm{CH})$, 
5.63(m,1H,-CH), 7.65(m, 1H, Ar-H), 7.87(m, 2H, Ar-H), 8.11(s, $1 \mathrm{H}, \quad$ Ar-H $), 8.39(\mathrm{~m}, 1 \mathrm{H}, \quad \mathrm{Ar}-\mathrm{H}) ;{ }^{13} \mathrm{C}$ NMR (100 MHz $\left.\mathrm{CDCl}_{3}\right): 12.0,18.7,20.8,23.1,25.4,28.4,30.1$, 31.3, 39.2, 42.4, 48.7, 50.2, 81.5, 124.2, 126.1, 126.5, 127.3, 128.1, 144.6, 149.5, 163.2; MS (ESI +) $m / z$ : calculated for $\mathrm{C}_{29} \mathrm{H}_{38} \mathrm{~N}_{4} \mathrm{O}[\mathrm{M}+\mathrm{H}]^{+}$458.64; found, 459.39 .

3-Hydroxy-1,3,5(10)-estratrien-17-(phthalazine-1yl-h ydrazono) (13) Yellow solid, 0.75 g, Yield 66\%; mp: 167$169{ }^{\circ} \mathrm{C}$; IR $\left(\mathrm{KBr}, \mathrm{cm}^{-1}\right): 1585(\mathrm{C}=\mathrm{C}), 1602,1649(\mathrm{C}=\mathrm{N})$, 2935(CH), 3055(aromatic $\mathrm{CH}), 3383(\mathrm{NH}) ;{ }^{1} \mathrm{H}$ NMR (400 MHz DMSO) $\delta$, ppm: 0.98(s, 3H, $\left.-\mathrm{CH}_{3}\right), 6.51(\mathrm{~d}, 1 \mathrm{H}$, $J=4 \mathrm{~Hz}, \mathrm{Ar}-\mathrm{H}$, estrone), $6.57(\mathrm{~d}, 1 \mathrm{H}, J=4 \mathrm{~Hz}$, estrone), 7.14(d, $1 \mathrm{H}, J=8.48 \mathrm{~Hz}$, Ar- $\mathrm{H}$, estrone), 7.70 (m, 3H, Ar- $-\mathrm{H}$, hydralazine), $8.00(\mathrm{~s}, 1 \mathrm{H}, \mathrm{Ar}-\mathrm{H}$, hydralazine), 8.23(s, $1 \mathrm{H}, \mathrm{Ar}-\mathrm{H}$, hydralazine), 9.08(s, $1 \mathrm{H},-\mathrm{OH}), 11.21(\mathrm{~s}$, $1 \mathrm{H},-\mathrm{NH}) ;{ }^{13} \mathrm{C}$ NMR (100 MHz DMSO): 16.9, 25.9, 27.2, 29.1, 30.7, 35.1, 38.1,43.8, 44.3, 52.1, 112.86, 114.9, 115.9, 123.3, 126.2, 126.8, 131.4, 136.5, 137.1, 145.7, 168.0, 176.6; MS (ESI+) $m / z$ : Calculated for $\mathbf{C}_{26} \mathbf{H}_{28} \mathbf{N}_{4} \mathbf{O}[\mathrm{M}+\mathrm{H}]^{+}$ 412.53; found, 413.4. Anal. Calc. C:75.70, H:6.84, N:13.58; found (C:74.35, H:6.72, N:13.59).

3-(Phthalazin-1yl-hydrazono)-4-methyl-19-nor-4-an drostene-17ß-ol (15) Yellow solid, $0.4 \mathrm{~g}$, Yield 36\%; $\mathrm{mp}:>200{ }^{\circ} \mathrm{C}$; IR $\left(\mathrm{KBr}, \mathrm{cm}^{-1}\right): 1579(\mathrm{C}=\mathrm{C}), 1607(\mathrm{C}=\mathrm{N})$, 2932(CH), 3366 (NH), 3415(OH); ${ }^{1}$ HNMR (400 MHz $\left.\mathrm{CDCl}_{3}\right) \delta$, ppm: 0.93(s, $\left.3 \mathrm{H},-\mathrm{CH}_{3}\right), 1.22\left(\mathrm{~s}, 3 \mathrm{H},-\mathrm{CH}_{3}\right)$, $3.53(\mathrm{~s}, 1 \mathrm{H},-\mathrm{CH}), 7.71(\mathrm{~m}, 1 \mathrm{H}, \mathrm{Ar}-\mathrm{H}), 7.87(\mathrm{~m}, 2 \mathrm{H}$, $\mathrm{Ar}-\mathrm{H}), 8.18(\mathrm{~s}, 1 \mathrm{H}, \mathrm{Ar}-\mathrm{H}), 8.51(\mathrm{~m}, 5 \mathrm{H}, \mathrm{Ar}-\mathrm{H}) ;{ }^{13} \mathrm{C}$ NMR $\left(100 \mathrm{MHz} \mathrm{CDCl}_{3}\right): 11.3,18.0,23.3,24.7,26.1,26.5,29.7$, 30.1, 30.6, 35.4, 36.4, 40.4, 42.5, 43.0, 49.5, 49.7, 81.5, 124.4, 129.2, 138.4, 166.9; MS (ESI+) $m / z$ : Calculated for $\mathrm{C}_{27} \mathrm{H}_{34} \mathrm{~N}_{4} \mathrm{O}[\mathrm{M}+\mathrm{H}]^{+}$430.27; found, 431.18.

3-(Phthalazin-1yl-hydrazono)-4,4'-dimethyl-19-nor -5-androstene-17 $\beta$-ol (16) Yellow solid, $0.6 \mathrm{~g}$, Yield 66.6\%; mp: $109-108{ }^{\circ} \mathrm{C}$; IR $\left(\mathrm{KBr}, \mathrm{cm}^{-1}\right)$ : $1588(\mathrm{C}=\mathrm{C})$, 1606 $(\mathrm{C}=\mathrm{N}), 2954(\mathrm{CH}), 3371(\mathrm{NH}), 3456(\mathrm{OH}) ;{ }^{1} \mathrm{H}$ NMR $\left(400 \mathrm{MHz} \mathrm{CDCl}_{3}\right) \delta$, ppm: 0.97(s, 3H, $\left.-\mathrm{CH}_{3}\right), 1.21(\mathrm{~s}, 6 \mathrm{H}$, $\left.-\mathrm{CH}_{3}\right), 3.57(\mathrm{~s}, 1 \mathrm{H},-\mathrm{CH}), 5.56(\mathrm{~d}, 1 \mathrm{H}, J=7.1 \mathrm{~Hz},-\mathrm{CH})$, 7.51(m, 1H, Ar-H), 7.71(m, 2H, Ar-H), 8.21(s,1H,Ar-H), 8.41(m, $1 \mathrm{H}, \mathrm{Ar}-\mathrm{H}) ;{ }^{13} \mathrm{C}$ NMR $\left(100 \mathrm{MHz} \mathrm{CDCl}_{3}\right)$ : 13.6, 21.4, 23.9, 24.7, 27.4, 30.7, 31.1, 31.8, 32.1, 33.8, 37.1, 38.4, 44.2, 48.9, 80.8, 119.9, 124.3, 124.6, 125.5, 127.0, 127.4, 128.3, 131.2, 131.8, 133.4, 137.9, 146.6, 152.3, 162.3; MS $(\mathrm{ESI}+) \mathrm{m} / z$ : calculated for $\mathbf{C}_{28} \mathbf{H}_{\mathbf{3 8}} \mathbf{N}_{\mathbf{4}} \mathbf{O}[\mathrm{M}+\mathrm{H}]^{+} 446.63$; found, 447.47.

17-Ethinyl-3-(phthalazin-1yl-hydrazono)-estr-4,6-d ien-17ß-ol (18) Yellow solid, 0.55 g, Yield 74\%; mp: $167-169{ }^{\circ} \mathrm{C}$; IR $\left(\mathrm{KBr}, \mathrm{cm}^{-1}\right): 1582(\mathrm{C}=\mathrm{C}), 1619(\mathrm{C}=\mathrm{N})$, 2977(CH), 3322(NH), $3401(\mathrm{OH}) ;{ }^{1} \mathrm{H}$ NMR $(400 \mathrm{MHz}$
$\left.\mathrm{CDCl}_{3}\right) \delta$, ppm: 0.95(s,3H, $\left.-\mathrm{CH}_{3}\right), 2.86(\mathrm{~s}, 1 \mathrm{H}, \mathrm{CH}), 5.80(\mathrm{~d}$, $1 \mathrm{H}, J=10.7 \mathrm{~Hz}, \mathrm{CH}), 5.92(\mathrm{~s}, 1 \mathrm{H},-\mathrm{CH}), 6.23(\mathrm{~d}, 1 \mathrm{H}$, $J=10.7 \mathrm{~Hz},-\mathrm{CH}), 7.60(\mathrm{~m}, 1 \mathrm{H}, \mathrm{Ar}-\mathrm{H}), 7.79(\mathrm{~m}, 2 \mathrm{H}, \mathrm{Ar}-\mathrm{H})$, 8.28(s,1H,Ar-H), 8.43(m,1H,Ar-H); ${ }^{13} \mathrm{C}$ NMR $(100 \mathrm{MHz}$ $\left.\mathrm{CDCl}_{3}\right): 12.0,22.5,25.1,26.9,32.3,37.8,38.7,40.8,41.9$, 45.7, 47.3, 48.7, 74.3, 80.4, 87.0, 124.4, 125.6, 126.9,127.5, 128.8, 131.6, 132.1, 136.5, 141.5, 158.9, 161.8; MS (ESI +) $m / z$ : Calculated for $\mathbf{C}_{28} \mathbf{H}_{30} \mathbf{N}_{4} \mathbf{O}[\mathrm{M}+\mathrm{H}]^{+}$; 438.56; found, 439.49 .

3-(Phthalazin-1yl-hydrazono)-1 $\alpha$-methyl-5 $\alpha$-an drostan-17ß-ol (20) Yellow solid, $0.67 \mathrm{~g}$, Yield 61\%; $\mathrm{mp}:>200 \quad{ }^{\circ} \mathrm{C}$; $\quad$ IR $\quad\left(\mathrm{KBr}, \quad \mathrm{cm}^{-1}\right): 1586(\mathrm{C}=\mathrm{C})$, $1629(\mathrm{C}=\mathrm{N}), 2930(\mathrm{CH}), 3392(\mathrm{OH}) ;{ }^{1} \mathrm{H}$ NMR $(300 \mathrm{MHz}$ $\left.\mathrm{CDCl}_{3}\right) \quad \delta$, ppm: $0.74-1.0(\mathrm{~m}, 14 \mathrm{H}$, Mesterolone $)$, $1.0-1.79(\mathrm{~m}, 11 \mathrm{H}$, Mesterolone), $3.58(\mathrm{t}, 1 \mathrm{H}, J=9.0 \mathrm{~Hz},-$ $\mathrm{CH}), 7.40-7.45(\mathrm{~m}, 1 \mathrm{H}, \mathrm{Ar}-\mathrm{H}), 7.56-7.59(\mathrm{~m}, 2 \mathrm{H}, \mathrm{Ar}-\mathrm{H})$, $7.72(\mathrm{~d}, 1 \mathrm{H}, J=6 \mathrm{~Hz}, \mathrm{Ar}-\mathrm{H}), 8.30-8.35(\mathrm{~m}, 1 \mathrm{H}, \mathrm{Ar}-\mathrm{H}){ }^{13} \mathrm{C}$ NMR (75 MHz CDCl $)_{3}$ ): 11.3, 14.0, 14.8, 20.0, 23.5, 28.7, 30.5 , 30.9, 31.3, 35.6, 36.7, 38.6, 38.9, 39.2, 39.6, 40.6, 43.1, 48.7, 48.9, 51.0, 81.8, 124.1, 126.0, 127.2, 131.4, 131.6, 137.4, 146.4, 166.8; MS (ESI+) $m / z$ : Calculated for $\mathrm{C}_{28} \mathrm{H}_{38} \mathrm{~N}_{4} \mathrm{O}[\mathrm{M}+\mathrm{H}]^{+}$446.63; found, 447.92.

4'-[17-(Phthalazin-1-yl-hydrazone)-1,3,5(10)-estratri en-3-yloxymethyl]-biphenyl-2-carbonitrile (22) Yellow solid, $0.50 \mathrm{~g}$, Yield $76.9 \%$; mp: $>200{ }^{\circ} \mathrm{C}$; IR $(\mathrm{KBr}$, $\left.\mathrm{cm}^{-1}\right): \quad 1586(\mathrm{C}=\mathrm{C}), \quad 1602(\mathrm{C}=\mathrm{N}), \quad 1648, \quad 2223(\mathrm{C} \equiv \mathrm{N})$, 2937 $(\mathrm{CH}), \quad 3064\left(\mathrm{CH}\right.$ aromatic), 3398(NH); ${ }^{1} \mathrm{H}$ NMR $\left(400 \mathrm{MHz} \mathrm{CDCl}_{3}\right) \delta$, ppm: 1.1(s, 3H, $\left.-\mathrm{CH}_{3}\right), 5.16(\mathrm{~s}, 2 \mathrm{H},-$ $\left.\mathrm{OCH}_{2}-\right), 6.80-8.85(\mathrm{~m}, 20 \mathrm{H}, \mathrm{Ar}-\mathrm{H}) ;{ }^{13} \mathrm{C}$ NMR $(100 \mathrm{MHz}$ $\left.\mathrm{CDCl}_{3}\right): 17.0,25.1,27.3,29.1,30.7,35.1,38.1,43.8,44.3$, 52.1, 112.86, 114.9, 115.9, 123.3, 126.2, 126.8, 131.4, 136.5, 137.1, 137.8, 141.4, 145.7, 149.7, 154.0, 167.1, 183.5; MS (ESI+) $m / z$ : calculated for $\mathbf{C}_{40} \mathbf{H}_{37} \mathbf{N}_{5} \mathbf{O}[\mathrm{M}+\mathrm{H}]^{+}$603.75; found, 604.86 .

\section{Discussion}

\section{Chemistry}

The literature survey was done by focusing on steroidal hydrazone where ketosteroid used as a starting material. Allah HMF synthesized phthalazinohydrazone of $17 \alpha$-methyltestosterone [33]. Rasras et al. [34] conveyed the efficient procedure for the synthesis of novel hydrazide-hydrazone of cholic acid and tested them for antibacterial activity. Mohareb et al. reported synthesis of hydrazide-hydrazone, pyrazole, pyridine, thiazole, thiophene derivatives and their cytotoxicity evaluations [35]. A method conveyed by Nadaria et al. [36, 37] for the synthesis and biological activity of hydrazone of $5 \alpha$-steroids and synthesis and cytotoxicity of epiandrosterone hydrazones. Jaben et al. [38] specified the synthesis of 
anticancer agents of progesterone and testosterone. Zickovic et al. [39] synthesized steroidal thiosemicarbazones and evaluated their cytotoxic activity.

The structural chemistry of these steroidal hydrazones involves the condensation of hydralazine hydrochloride at $\mathrm{C} 3$ and $\mathrm{C} 17$ of ketosteroid. The reaction is catalyzed by potassium acetate in methanol as solvent. Androgen and estrogen scaffold used as ketosteroid for the synthesis of title compounds. The synthesis of steroidal hydrazones was developed without chromatographic purification (column/flash chromatography).

The structures of the synthesized compounds (3-8, $10,11,15,16,18,20$ and 22) were established using ${ }^{1} \mathrm{H}$, ${ }^{13} \mathrm{C}$-NMR and mass spectral data. In ${ }^{1} \mathrm{H}$ NMR spectrum of steroidal hydrazones $(3-8,10,11,15,16,18$, 20 and 22) singlet signals of $4-\mathrm{CH}_{3}, 4,4^{\prime}-\mathrm{CH}_{3}, 18-\mathrm{CH}_{3}$ and $19-\mathrm{CH}_{3}$ groups were present at $\delta 1.22 \mathrm{ppm}, 0.76-$ $0.96 \mathrm{ppm}$ and $0.81 \mathrm{ppm}$ and doublet signal of $7-\mathrm{CH}_{3}$ group was present at $\delta 0.73-0.75 \mathrm{ppm}$. Aromatic protons of hydralazine were noted in the interval at 87.5-8.5 ppm. In the ${ }^{13} \mathrm{C}$ NMR spectra of steroidal hydrazones $(5,6$ and 18$)$ peaks of $\equiv \mathrm{CH}$ carbon existence at around $\delta 79.0 \mathrm{ppm}$ and $-\mathrm{C} \equiv$ at around $87 \mathrm{ppm}$. Signals of $\mathrm{C}=\mathrm{N}$ bond at $161.6 \mathrm{ppm}$ and $168.0 \mathrm{ppm}$. The C17 peaks of steroidal hydrazones $(3-8,10,11,15,16$, 18,20 and 22) were observed at around $\delta 81 \mathrm{ppm}$. In ${ }^{1} \mathrm{H}$ NMR spectrum of steroidal hydrazones $(3-8,11$, 15,16 and 18) and singlet signal of $-\mathrm{CH}$ were present at around $\delta 2.48-3.61 \mathrm{ppm}$. Where as in compound 10 and $20,-\mathrm{CH}$ gave triplet at $\delta 3.55$ and $3.58 \mathrm{ppm}$. In the mass spectral analysis $[\mathrm{M}+\mathrm{H}]^{+}$of $9 \mathrm{~A}, 14 \mathrm{~A}, 14 \mathrm{~B}, 10,13$ and 20 matched with theoretical values.

In the ${ }^{1} \mathrm{H}$ NMR spectra (in DMSO) of steroidal hydrazone (13) singlet signals of angular $18-\mathrm{CH}_{3}$ group was present at $\delta 0.98 \mathrm{ppm}$. The signals of aromatic protons were present in the range of $\delta 6.57-7.14 \mathrm{ppm}$. The singles of aromatic protons of hydralazine were present in the range of $\delta 7.70-8.23 \mathrm{ppm}$. Singlet signal of the proton of $-\mathrm{OH}$ group at $\delta 9.08 \mathrm{ppm}$. The protons of the $-\mathrm{NH}$ at $\delta 11.21 \mathrm{ppm}$. The IR spectrum of the steroidal hydrazone (13) contained absorption bands the $\mathrm{NH}$ - group at $3358 \mathrm{~cm}^{-1}, \mathrm{C}=\mathrm{N}$ bond at $1649 \mathrm{~cm}^{-1}$, bands at 1602 , $1585 \mathrm{~cm}^{-1}$ for $-\mathrm{C}=\mathrm{C}-$. The infrared spectra of steroidal hydrazones $(3-8,10,11,15,16,18,20$ and 22$)$ showed the $\mathrm{NH}$-band in the range of $3381-3209 \mathrm{~cm}^{-1}$.

\section{Biological activity In vitro antimicrobial activity}

We have selected compounds 3 (testosterone), 6 (iso tibolone), 7 (7-methyl nandrolone), 10 (86-testosterone), 11 (4,4-dimethyl steroid) and 18 ( $\delta 6$-norethisterone) for the antimicrobial screening based on the steroidal skeleton.
Table 1 Antibacterial activity of steroidal hydrazones

\begin{tabular}{lllll}
\hline Compounds & $\begin{array}{l}\text { E. coli } \\
\text { MTCC 443 }\end{array}$ & $\begin{array}{l}\text { P. aeruginosa } \\
\text { MTCC 1688 }\end{array}$ & $\begin{array}{l}\text { S. aureus } \\
\text { MTCC 96 }\end{array}$ & $\begin{array}{l}\text { S. pyogenus } \\
\text { MTCC 442 }\end{array}$ \\
\hline \multicolumn{4}{l}{ MIC (minimal inhibition concentration) $\mu$ mol } \\
3 & 580 & 1159 & 232 & 1345 \\
6 & 219 & 110 & 549 & 55 \\
7 & 145 & 231 & 289 & 1159 \\
10 & 116 & 582 & 116 & 582 \\
11 & 272 & 142 & 544 & 217 \\
18 & 568 & 284 & 227 & 1137 \\
Gentamycin & 0.1 & 2 & 0.5 & 1 \\
Ampicillin & 286 & 286 & 715 & 286 \\
Chloramphenicol & 154 & 154 & 154 & 154 \\
Ciprofloxacin & 75 & 75 & 150 & 150 \\
Norfloxacin & 31 & 31 & 31 & 31 \\
\hline
\end{tabular}

The difference in the structure of above compounds is the presence of $-\mathrm{CH}_{3}$, Unsaturation and ethinyl group. The position of groups are encourages us that what will be the activity of the selected compounds among the all.

The in vitro antimicrobial activity of some of the synthesized compounds was accomplished by broth microdilution method [40]. It is one of the non-automated in vitro bacterial susceptibility tests. This classic method yields a quantitative result for the amount of antimicrobial agents that is needed to inhibit growth of specific microorganisms. It is carried out in tubes. Mueller-Hinton broth was used as nutrient medium to grow and dilute the compound suspension for the test bacteria and Sabouraud Dextrose broth used for fungal nutrition.

Each synthesized drug was diluted obtaining $2000 \mu \mathrm{g} /$ $\mathrm{ml}$ concentration, as a stock solution.

Primary screen In primary screening $1000 \mathrm{micro} / \mathrm{ml}$, $500 \mathrm{micro} / \mathrm{ml}$ and $250 \mathrm{micro} / \mathrm{ml}$ concentrations of the synthesized drugs were taken. The active synthesized drugs found in this primary screening were further tested in a second set of dilution against all microorganisms.

Secondary screen The drugs found active in primary screening were similarly diluted to obtain $200 \mathrm{micro} / \mathrm{ml}$, $100 \mathrm{micro} / \mathrm{ml}, 50 \mathrm{micro} / \mathrm{ml}, 25 \mathrm{micro} / \mathrm{ml}, 12.5 \mathrm{micro} /$ $\mathrm{ml}, 6.250 \mathrm{micro} / \mathrm{ml}$ and concentrations.

Reading result The highest dilution showing at least 99\% inhibition zone is taken as MIC. The result of this is much affected by the size of the inoculum. The test mixture should contain $10^{8}$ organism $/ \mathrm{ml}$. Inoculum size for test strain was adjusted to $10^{8} \mathrm{CFU}$ [Colony Forming Unit] per milliliter by comparing the turbidity. The 
strains employed for the activity were procured from [MTCC-Micro Type Culture Collection] Institute of Microbial Technology, Chandigarh.

The compounds $3,6,7,10,11$ and 18 were screened for their antibacterial activity against Escherichia coli (E. coli), Pseudomonas aeruginosa ( $P$. aeruginosa), Staphylococcus aureus (S.aureus), Streptococcus pyogenes (S. pyogenes) and antifungal activity against Candida albicans (C. albicans), Aspergillus niger (A. Niger) and Aspergillus clavatus (A. Clavatus). DMSO was used as media to get desired concentration of compounds to test upon microbial strains. The lowest concentration, which showed no visible growth after spot subculture was considered as MIC for each compound. The standard antibiotics used for comparison in the present study were gentamycin, ampicillin, chloramphenicol, ciprofloxacin and norfloxacin for evaluating antibacterial activity while nystatin and griseofulvin for antifungal activity. The results are summarized in Tables 1 and 2.

From the antimicrobial data, steroidal hydrazones 3, 7, 10 and 18 showed better activity (MIC 116-289 $\mu \mathrm{M}$ ) against gram-positive bacteria Staphylococcus aureus ( $S$. aureus) as compare to ampicillin (MIC $715 \mu \mathrm{M}$ ). Compounds 7 and 10 showed excellent activity (MIC 145 and $116 \mu \mathrm{M})$ against gram-negative bacteria Escherichia coli (E. coli) as compared to ampicillin (MIC $286 \mu \mathrm{M}$ ), compound 6 (MIC $55 \mu \mathrm{M}$ ) is found active against Streptococcus pyogenes (S. pyogenes) as compare to chloramphenicol (MIC 154 $\mu \mathrm{M}$ ) and ciprofloxacin (MIC $150 \mu \mathrm{M}$ ). Compound 6 (MIC $110 \mu \mathrm{M}$ ) and 11 (MIC $142 \mu \mathrm{M}$ ) exhibited powerful activity against Pseudomonas aeruginosa (P. aeruginosa) as compare to ampicillin (MIC $286 \mu \mathrm{M}$ ). Compound 6 showed equivalent potency against Escherichia coli (E. coli) and Staphylococcus aureus (S. aureus) as compared to ampicillin.

Table 2 Antifungal activity of steroidal hydrazones

\begin{tabular}{lccc}
\hline Compounds & $\begin{array}{l}\text { C. albicans } \\
\text { MTCC 227 }\end{array}$ & $\begin{array}{l}\text { A. niger } \\
\text { MTCC 282 }\end{array}$ & $\begin{array}{l}\text { A. clavatus } \\
\text { MTCC 1323 }\end{array}$ \\
\hline MIC (minimal inhibition concentration) & (mol & \\
3 & 1159 & 1159 & 2319 \\
6 & 2196 & 2196 & 2196 \\
7 & 580 & 580 & 2319 \\
10 & 1164 & 1164 & $>1164$ \\
11 & 1088 & 1088 & 1088 \\
18 & 1137 & 2274 & $>2274$ \\
Nystatin & 108 & 108 & 108 \\
Griseofulvin & 1417 & 283 & 283 \\
\hline
\end{tabular}

Entire steroidal hydrazones (MIC 580-2319 $\mu \mathrm{M}$ ) showed inferior activity against all gram-positive and gram-negative bacteria as compare to gentamycin (MIC $0.10-2 \mu \mathrm{M})$ and norfloxacin (MIC $31 \mu \mathrm{M}$ ). Compound $7(\mathrm{MIC} 580 \mu \mathrm{M})$ is found active against C. albicans as compare to griseofulvin (MIC $1417 \mu \mathrm{M}$ ) and rest of the steroidal hydrazones exhibited less potency than standard fungicidal nystatin and griseofulvin against Candida albicans, Aspergillus niger and Aspergillus clavatus.

\section{Conclusions}

The antimicrobial activities of steroidal hydrazone were studied by the broth microdilution method. Compound 6, 7 and 10 displayed excellent antibacterial activity among the tested compounds due to bearing an ethinyl at $\mathrm{C}-17$ of compound 6, methyl at C-7 of compound 7. Compound 10 showed excellent antibacterial activity due to the compounds bearing an additional $-\mathrm{C}=\mathrm{C}-$ in the structure. Hence, these substituted steroidal skeleton considered for the development of the new antimicrobial agent.

\section{Acknowledgements \\ We are thankful to D. K. Pharma, Powoi, Mumbai for generous gift of hydrala- zine hydrochloride.}

\section{Authors' contributions}

SM contributed to synthesis, characterization and activity. AKS contributed to analytical work. All authors have read and approved the manuscript.

\section{Funding}

No funding was received for this work.

Availability of data and materials

Data and materials are available upon request.

\section{Declarations}

Ethics approval and consent to participate

Not applicable.

\section{Consent for publication \\ Not applicable.}

\section{Competing interests}

The authors declare that they have no competing interests.

Received: 1 June 2021 Accepted: 22 December 2021

Published online: 06 January 2022

References

1. Rollas S, Kucukguzel SG (2007) Biological activities of hydrazone derivatives. Molecules 12:1910-1939

2. Subhashini NJP, Janaki P, Bhadraiah B (2017) Synthesis of hydrazone derivatives of benzofuran and their antibacterial and antifungal activity. Russ J Gen Chem 87:2021-2026

3. Sridhar KS, Pandeya SN, Stables JP, Atmakuru R (2002) Anticonvulsant activity of hydrazones, Schiff and Mannich bases of isatin derivatives. Eur J Pharm Sci 16:129-132 
4. Debnatha U, Mukherjee S, Joardar N, Sinha BS, JanaMisra KAK (2019) Aryl quinolinyl hydrazone derivatives as anti-inflammatory agents that inhibit TLR4 activation in the macrophages. Eur J Pharm Sci 134:102-115

5. Kumar P, Kadyan K, Duhan M, Sindhu J, Singh V, Singh B, Kumar S (2017) Design, synthesis, conformational and molecular docking study of some novel acyl hydrazone based molecular hybrids as antimalarial and antimicrobial agents. Chem Cent J 11:115

6. Nogueira TM, Cruz LS, Lourenço M, Nora de Souza MV (2019) Design, synthesis and anti-tuberculosis activity of hydrazones and $\mathrm{N}$-acylhydrazones containing vitamin b6 and different heteroaromatic nucleus. Lett Drug Des Discov 16:7

7. Singh V, Srivastava VK, Palit G, Shanker K (1992) Coumarin congeners as antidepressants. Arzneim-Forsch Drug Res 42(8):993-996

8. Gan C, Liu L, Cui J, Liu Z, Shi H, Lin Q, Sheng H, Yang C, Huang Y (2017) Synthesis of some steroidal derivatives with side chain of 20 - and 22-hydrazone aromatic hetero cycles and their antiproliferative activity. Med Chem 13(4):375-383

9. Loncle C, Brunel JM, Vidal N, Dherbomez M, Letourneux Y (2004) Synthesis and antifungal activity of cholesterol-hydrazone derivatives. Eur J Med Chem 39:1067-1071

10. Visbal G, San-Blas G, Maldonado A, Alvarez-Aular A, Capparelli MV, Murgich J (2011) Synthesis, in vitro antifungal activity and mechanism of action of four sterol hydrazone analogues against the dimorphic fungus Paracoccidioides brasiliensis. Steroids 76:1069-1081

11. Khan SA, Kumar P, Joshi R, lqbal PF, Saleem K (2008) Synthesis and in vitro antibacterial activity of new steroidal thiosemicarbazone derivatives. Eur J Med Chem 43(9):2029-2034

12. Gan C, Cui J, Su S, Lin Q, Jia L, Fan L, Huang Y (2014) Synthesis and antiproliferative activity of some steroidal thiosemicarbazones, semicarbazones and hydrozones. Steroids 87:99-107

13. Merlani MI, Kemertelidze EP, Papadopoulos K, Men'shova NI (2004) Some derivatives of 5 a ketosteroid hydrazones: synthesis from tigogenin and antituberculosis activity. Russ J Bioorgan Chem 30(5):497-501

14. Nadaraia NS, Onashvili EO, Kakhabrishvili ML, Barbakadze NN, Sylla B, Pichette A (2016) Synthesis and antiviral activity of several $N$-containing 5a-steroids. Chem Nat Compd 52(5):853-855

15. Nadaraia NS, Barbakadze NN, Kakhabrishvili ML, Sylla B, Pichette A, Makhmudov US (2018) Synthesis and biological activity of several modified 5a-androstanolone derivatives. Chem Nat Compd 54(2):310-314

16. Wang HJ, Bu M, Wang J, Liu L, Zhang S (2019) Synthesis and biological evaluation of novel steroidal $5 a$, 8a-endoperoxide steroidal derivatives with aromatic hydrazone side chain as potential anticancer agents. Russ J Bioorg Chem 45:585-590

17. Dhingra N, Bhardwaj TR, Mehta N, Mukhopadhyay T, Kumar A, Kumar $M$ (2010) Synthesis, antiproliferative, acute toxicity and assessment of antiandrogenic activities of some newly synthesized steroidal lactams. Eur J Med Chem 45:2229-2236

18. Huang Y, Cui J, Zheng Q, Zeng C, Chen Q, Zhou A (2012) 6-Hydroximino4-aza-Ahomo-cholest-3-one and related analogue as a potent introducer of apoptosis in cancer cells. Steroids 77:829-834

19. Duha CY, Loa IW, Wang SK, Dai CF (2007) New cytotoxic steroids from the soft coral Clavulariaviridis. Steroids 72:573-579

20. Malika IO, Maurice S (2006) Recent advances in thiasteroids chemistry. Steroids 71:1025-1044

21. Hanson JR (2006) Steroids: partial synthesis in medicinal chemistry. Nat Prod Rep 23:100-107

22. Chen SJ, Cui JG, Li Y, Fan LH (2011) Recent advance of steroidal hydrazone with biological activities. Chin J Org Chem 31(2):187-192

23. Cui J, Liu L, Zhao D, Gan C, Huang X, Xiao Q, Qi B, Yang L, Huang Y (2015) Synthesis, characterization and antitumor activities of some steroidal derivatives with side chain of 17-hydrazone aromatic heterocycle. Steroids 95:32-38

24. Cui JG, Liu L, Gan CF, Xiao Q (2014) Synthesis and biological activity of steroids bearing aromatic rings and heterocycles. Prog Chem 26(2/3):320-333

25. Xu H, Su X, Liu XQ, Zhang KP, Hou Z, Guo C (2019) Design, synthesis and biological evaluation of novel semicarbazone-selenochroman-4-ones hybrids as potent antifungal agents. Bioorg Med Chem Lett 29:126726

26. Stulov SV, Misharin AYu (2013) Synthesis of steroids with nitrogencontaining substituent's in ring D. Chem Heterocycl Compd 48(10):1431-1472
27. Li C, Qiu W, Yang Z, Luo J, Yang F, Liu M, Xie J, Tang J (2010) Stereoselective synthesis of some methyl-substituted steroid hormones and their in vitro cytotoxic activity against human gastric cancer cell line MGC-803. Steroids 75:859-869

28. Schroder NA (1952) The effect of 1-hydrasinophthalasine in hypertension. Circulation 5(1):28-37

29. Sousa C, Freire C, De Castro B (2003) Synthesis and characterization of benzo-15-crown-5 ethers with appended $\mathrm{N}_{2} \mathrm{O}$ Schiff bases. Molecules 8:894-900

30. Kajal A, Bala S, Kamboj S, Saini V (2014) Synthesis, characterization, and computational studies on phthalic anhydride-based benzylidenehydrazide derivatives as novel, potential anti-inflammatory agents. Med Chem Res 23:2676-2689

31. Cikla P, Tatar E, Kucukguzel I, Sahin F, Yurdakul D, Basu A, Krishnan R, KNicholsKaushik-BasuKucukguzel DBNSG (2013) Synthesis and characterization of flurbiprofen hydrazide derivatives as potential anti-HCV, anticancer and antimicrobial agents. Med Chem Res 22:5685-5699

32. Bedia KK, Oruc-Emre EE, Unsalan S, Rollas S (2009) Synthesis and anticancer activity of new hydrazide-hydrazones and their Pd(II) complexes. Med Chem Res 18:277-286

33. Allah HMF, Soliman R (1987) Synthesis and spectra of some triazolo and triazin phthalazines of possible hypotensive activity. J Hetero Cycl Chem 24:667-671

34. Rasras AJM, Al-Tel TH, Al-Aboudi AF, Al-Qawasmeh RA (2010) Synthesis and antimicrobial activity of cholic acid hydrazone analogues. Eur J Med Chem 45:2307-2313

35. Mohareb RM, Al-Omran F (2012) Novel synthesis of hydrazide-hydrazone, pyrazole, pyridine, thiazole, thiophene derivatives and their cytotoxicity evaluations. Steroids 77:1551-1559

36. Nadaraia NS, Barbakadze NN, Kakhabrishvili ML, Mshvildadze VD (2019) Synthesis and biological activity of hydrazones of $5 a$-steroids. Res J Pharm Biol Chem Sci 10(1):238-242

37. Nadaraia NS, Barbakadze NN, Mshvildadze VD, Sylla B, Legault J, Pichette A (2020) Synthesis and cytotoxicity of epiandrosterone hydrazones. Chem Nat Compd 56(2):274-277

38. Muafia J, Muhammad IC, Ghulam AM, Khondaker MR, Umer R, Khan HU, Arshiab F, Abdul S (2018) Synthesis, pharmacological evaluation and docking studies of progesterone and testosterone derivatives as anticancer agents. Steroids 136:22-31

39. Zivkovic MB, Matic IZ, Rodic MV, Novakovic IT, Sladic DM, Krstic NM (2016) Synthesis, characterization and in vitro cytotoxic activities of new steroidal thiosemicarbazones and thiadiazolines. RSC Adv 6:34312-34333

40. Wiegand I, Hilpert K, Hancock REW (2008) Agar and broth dilution methods to determine the minimal inhibitory concentration (MIC) of antimicrobial substances. Nat Protoc 3(2):165

\section{Publisher's Note}

Springer Nature remains neutral with regard to jurisdictional claims in published maps and institutional affiliations.

\section{Submit your manuscript to a SpringerOpen ${ }^{\circ}$ journal and benefit from:}

- Convenient online submission

- Rigorous peer review

- Open access: articles freely available online

- High visibility within the field

Retaining the copyright to your article

Submit your next manuscript at springeropen.com 